\title{
A randomised trial of weekend and evening breast screening appointments
}

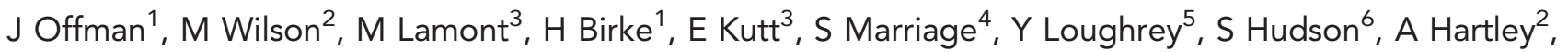
J Smith ${ }^{3}$, B Eckersley ${ }^{7}$, F Dungey ${ }^{8,9}$, D Parmar ${ }^{1}$, J Patnick ${ }^{10}$ and S W Duffy ${ }^{\star}, 1$

${ }^{1}$ Centre for Cancer Prevention, Wolfson Institute of Preventive Medicine, Barts and the London School of Medicine and Dentistry, Queen Mary, University of London, Charterhouse Square, London EC1M 6BQ, UK; ${ }^{2}$ University Hospital of South Manchester NHS Foundation Trust, The Nightingale Centre and Genesis Prevention Centre, Wythenshawe Hospital, Manchester, UK; ${ }^{3}$ University Hospital Bristol NHS Foundation Trust, Avon Breast Screening Unit, Central Health Clinic, Bristol, UK; ${ }^{4}$ University Hospital Bristol NHS Foundation Trust, Bristol Bath and Weston Abdominal Aortic Aneurysm Screening Programme, Bristol Royal Infirmary, Bristol, UK; ${ }^{5}$ Training Department, Liverpool Heart and Chest Hospital NHS Foundation Trust, Liverpool, UK; ${ }^{6}$ Peel and Schriek Consulting Ltd., London, UK; ${ }^{7}$ BMI The Alexandra Hospital, Cheadle, UK; ${ }^{8}$ PAREXEL International, Uxbridge, UK; ${ }^{9}$ Cancer Prevention Trials Unit, Queen Mary, University of London, London EC1M 6BQ, UK and ${ }^{10}$ NHS Cancer Screening Programmes, Sheffield, UK
\end{abstract}

Background: There is a need to research interventions that improve access to and convenience of breast cancer screening services.

Methods: We conducted a randomised trial comparing invitations to out-of-hours appointments with standard office hour appointments. Women who were to be invited for routine breast screening were randomised $(3: 1: 1: 1)$ to one of these screening invitations: standard office hour appointment, office hour appointment with the option to change to an out-of-hours appointment, weekday evening appointment, or weekend appointment.

Results: A total of 9410 women were invited to an office hour, 3519 to an office hour with the option to change, 3271 to a weekday evening, and 3162 to a weekend appointment. The offer of an initial out-of-hours appointment was associated with a nonsignificant decrease in attendance rates (73.7\% vs 74.1\%). The highest attendance was observed in the group offered an initial office hour appointment with the option to change to out-of-hours (76.1\% vs $73.3 \%$ for standard office hour, $P=0.001)$, with $7 \%$ of invitees exercising the option to change.

Conclusion: The optimum strategy for improving attendance at breast screening is to offer a traditional office hour appointment and including in the letter of invitation an option to change to an evening or weekend appointment if wished.

Although there is disagreement about the absolute size of the mortality reduction, it is generally accepted that mammography prevents deaths from breast cancer (Independent UK Panel on Breast Cancer Screening, 2012). The UK National Health Service (NHS) offers 3-year, two-view mammography to all women aged 50-70 years, which is currently being expanded to women aged 47-73 years in England. Although it must be accepted that a proportion of those offered screening will make an informed decision not to take up the offer, the provider of the service has a responsibility to ensure as far as possible that the decision is not unduly swayed by issues of convenience. Enhancing the accessibility of the programme is therefore desirable to improve the efficiency, effectiveness, and equity of the service.

Attendance in the UK NHS Breast Screening Programme exceeds $70 \%$ on average (NHS Cancer Screening Programmes, 2011). There are areas with considerably lower attendance rates though, and recent declines have been observed. Non-attendance for breast screening has been associated with various factors in 
United Kingdom and elsewhere, notably low socioeconomic status, specific health attitudes and beliefs, and distance from place of residence to the screening site (Maheswaran et al, 2006; Paskett et al, 2006; Rutter et al, 2006). Although one study in Spain found inconvenience of the appointment time as a reason for nonattendance (Alcaraz et al, 2002), the relationship of appointment time with attendance is not well researched. Preliminary surveys of American women found that women who worked felt that evening and weekend screening appointments would be useful (Engelman et al, 2005). This was mirrored in a previous survey of women in New Zealand, where $17 \%$ felt that offering out-of-hours appointments would increase attendance (Richardson, 1990). In contrast, a study in the 1990s suggested that offering appointments outside of working hours did not materially alter attendance rates (Readman and Asbury, 1999). However, with female employment rates higher now than in the past, the subject is worth revisiting.

It is furthermore not clear whether the offer of an evening appointment would, for example, be more welcome in summer than winter, or whether women would be happier attending an evening appointment in a hospital than in a mobile unit. In addition, location of a screening unit could have an impact on attendance of out-of-hours clinics, for example, a more centrally located screening unit might be more attractive on a Saturday, as the screening appointment could be combined with a shopping trip.

We carried out a randomised controlled study of offering appointments after working hours during the week and at weekends, with the aim to discover whether the offer of these out-of-hours screening appointments would increase attendance rates. To determine the optimum strategy for offering out-of-hours appointments, subgroup analysis was carried out to ascertain whether this effect is modified by prevalent or incident status, static or mobile screening sites, age of invitee, and time of year of appointment.

\section{MATERIALS AND METHODS}

Study design and participants. The Out of Hours Study (OOHS) was an open, four-arm (two major and four minor), randomised controlled trial undertaken at two breast screening centres in the United Kingdom.

The only eligibility criterion was appearance on the list of women (age 47-73 years) who were to be invited for their next routine breast screening appointment in the Greater Manchester Breast Screening Programme or in the Avon Breast Screening Programme in Bristol. Women were excluded from the study if they had opted out of the screening programme. At the beginning of this study, a second exclusion criterion had been defined as women who require a special appointment because of disability or breast implants. Once the study had started, however, it became apparent that it was not always possible to identify women requiring these special appointments at the time of randomisation. This exclusion criterion was therefore removed from the protocol, and women who had to reschedule their appointment due to access issues because of disabilities were removed from the data set before data analysis.

As this study represented only a minor variation on routine practice and some UK breast screening services are already offering out-of-hours appointments, women entered were not informed that they were subjects in a study or asked for consent. The study was approved by the National Research Ethics Service East London 3 Research Ethics Committee and the review boards of the participating organisations, and was carried out in accordance with good clinical practice guidelines and the Declaration of Helsinki. The study is registered as ISRCTN70398358.
Randomisation. Women who were to be invited for their next routine breast screening appointments were randomised to one of the four trial arms:

Major arm office hour appointments (arms (1) and (2)):

(1) Standard office hour appointment (without option to change to out-of-hours);

(2) Office hour appointment but with the invitation offering the opportunity to change to an evening or weekend appointment;

Major arm out-of-hours appointments (arms (3) and (4)):

(3) Weekday evening appointment; or

(4) Weekend appointment.

We planned to continue the study until we had recruited at least 9000 women to arm (1) and 3000 each to arms (2), (3), and (4), resulting in a ratio of approximately $3: 1: 1: 1$. Randomisation was by computerised pseudo-random number generation within the computerised breast screening invitation system for allocation to arms (3), (4), and arms (1) and (2) combined. The system could not be used to randomise between the two types of letters for arms (1) and (2), so a table of pseudo-random numbers was generated by STATA to allocate weeks of invitation between these two groups in the ratio $3: 1$.

Procedures. Women were sent the standard breast screening invitation letter normally used by the breast screening programme 2-4 weeks before their allocated appointment. In all groups, invitation letters stated explicitly that if the offered appointment was inconvenient, the screening unit would be happy to offer a different time and date.

Details of the first offered and any rescheduled appointments including reasons for rescheduling and appointment attendance were recorded. Women in all four trial arms were screened following the standard procedures.

Office hour appointments were made between 0845 and 1630 hours in Bristol and 0850 and 1630 hours in Manchester. Weekend appointments were on Saturdays within the same working hours normally used on weekdays. Weekday evening appointments were between 1700 and 1900 hours in Bristol and 1630 and 1900 hours in Manchester on at least 2 days per week, except Fridays.

The primary study end point was attendance for screening within 120 days of first offered appointment. The secondary end point was attendance at first offered appointment. The primary interest was in the difference in attendance between the two major arms, (1) and (2) combined compared with (3) and (4) combined, and between the minor arms within the major arms, (1) vs (2) and (3) vs (4).

Statistical analysis. The study was designed to have $90 \%$ power to detect as significant a difference in attendance of $60 \%$ vs $64 \%$ with 3000 women in each arm, and to considerably exceed this power in comparison of the 12000 with a standard appointment with the 6000 with an out-of-hours or weekend appointment.

In the context of this, study methods of handling withdrawals or protocol deviation were not applicable, because every woman received a letter ('treatment'). The statistical analysis was intentionto-treat, that is, the invitation group was allocated as randomised. However, the fact that there will be no deviation from the protocol implies that a per protocol analysis would be based on the same population, and therefore the results would be the same.

Statistical analysis was by logistic regression (Breslow and Day, 1980) with attendance as defined above as the end point, using conservative s.e. estimates (Gail et al, 1988) in case of any extrabinomial variation conferred by the randomisation of weeks between arms (1) and (2). In addition to the main comparisons of attendance between arms, a number of subgroup analyses were also planned by age, prevalent/incident screen status, whether previous 
scheduled screen (if applicable) had been attended, season of appointment, and type of screening unit (static or mobile). Analyses were performed using STATA Version 12.1 software (StataCorp, College Station, TX, USA). Season of appointment was coded as spring (March-May), summer (June-August), autumn (September-November), and winter (December-February). Prevalent/incident status was defined as incident if the woman had attended at least one previous screen and prevalent if not (regardless of the number of invitations). Statistical testing was two-sided, with $P<0.05$ considered to be statistically significant.

Role of the funding source. The funding source did not participate in the study design, data collection, data analysis, or drafting of the report. One author (JP) is directly employed by the funding source. SWD had full access to all the data in the study and had final responsibility for the decision to submit for publication.

\section{RESULTS}

Between June 2010 and July 2011, a total of 19409 women were enrolled into the OOHS. Forty-seven women were later excluded as they reported disabilities or access issues when re-booking. This resulted in 19362 women included in the trial, of whom 9930 were from the Greater Manchester Breast Screening Programme and 9432 were from the Avon Breast Screening Programme in Bristol. Figure 1 shows the CONSORT diagram for flow of the participants through the trial. A total of 9410 women were allocated to standard office hour appointments, 3519 to office hour appointments with option to change to out-of-hours, 3271 to evening, and 3162 to weekend appointments. Women were followed for 140 days after their first offered appointment, and the cut off date for appointment attendance was 120 days after their first offered appointment.

Table 1 shows the basic attributes of the study participants. The majority of women were aged $\geqslant 59$ years. In all, $27.0 \%$ of the screening episodes were prevalent screens, and $95.8 \%$ of screening invitations were to static sites. Also, $61.9 \%$ of appointments were in autumn or winter (defined as September-February inclusive). Of those women for whom this was the second or subsequent invitation, $76.9 \%$ had attended the previous screen.

Table 2 shows attendance within 120 days, attendance at first offered appointment, and numbers switching between out-ofhours and office hour appointments by trial arm for all the 19362 women entered into the study. The highest attendance (76.1\%) was observed for the arm offered an initial office hour appointment with the option to change to out-of-hours, compared with $73.3 \%$ in the standard office hour arm, $74.8 \%$ in the evening arm, and $72.6 \%$ in the weekend arm. Attendance differed significantly among all four arms $(P=0.001)$. In all, $7 \%$ of women in the office hour with option to change group took up the option to change to an out-ofhours appointment. Overall attendance was $73.9 \%$, but attendance at initial offered appointment was low (43.3\%).

From Figure 2A, it can be seen that there was no significant difference in attendance between offering office hour and out-ofhours appointments (the two major arms). The three out-of-hours study arms (office hour option to out-of-hours, evening, and weekend) were then compared with the standard invitation to an office hour appointment. Attendance was significantly higher for those whose invitation to an office hour appointment included the option to change to out-of-hours $(76.1 \%$ vs $73.3 \%$, odds ratio $(\mathrm{OR})=1.158, P=0.001)$. On the other hand, there was no statistically significant increase in attendance for initial evening or weekend appointments. Comparing the two initial out-of-hours appointments, evening $v s$ weekend, attendance was significantly lower in those offered a weekend appointment $(72.6 \%$ vs $74.8 \%$, $\mathrm{OR}=0.894, P=0.049$ ).

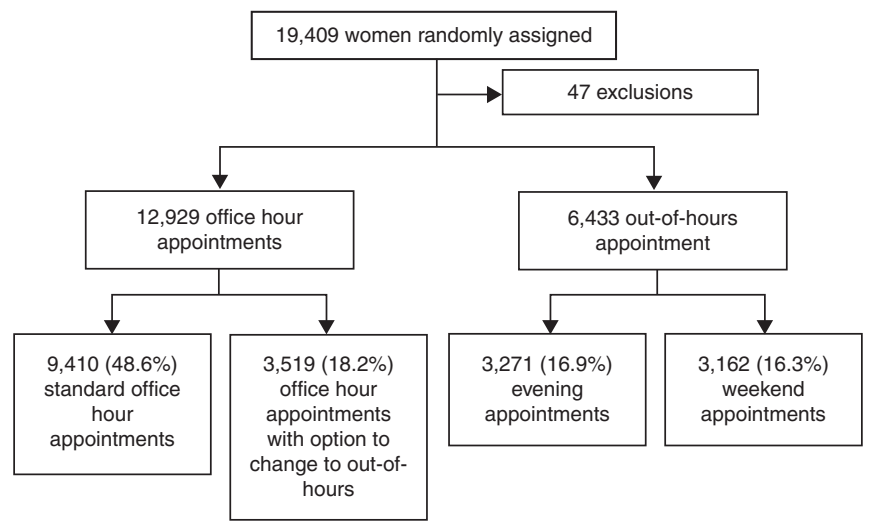

Figure 1. Basic design of the study and numbers of subjects randomised.

Table 1. Description of the trial population

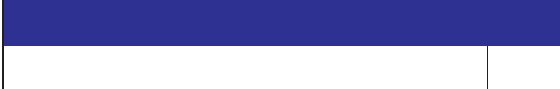

\begin{tabular}{l|l} 
n & $\%$
\end{tabular}

\section{Trial arm}

Standard office hours

Office hours with option to $\mathrm{OOH}$

Evening

Weekend

\begin{tabular}{l|l}
9410 & $48.6 \%$
\end{tabular}

$3519 \quad 18.2 \%$

3271

3162

$16.9 \%$

19362

$16.3 \%$

$100 \%$

Attendance at previous screen ( 3 years ago; only if previous invitations $\geqslant 1$ )

\section{Attended}

Not-attended ${ }^{a}$

12314

3710

$76.9 \%$

16024

$23.2 \%$

\section{Age group}

47 to $<54$

54 to $<59$

59 to $<65$

65 to $<74$

4514

4300

5298

5250

19362

$23.3 \%$

$22.2 \%$

$27.4 \%$

$27.1 \%$

$100 \%$

\section{Prevalent/incident status}

Prevalent

Incident

5232

14130

19362

$27.0 \%$

$73.0 \%$

$100 \%$

Screening site

\section{Static}

Mobile

18549

$95.8 \%$

813

$4.2 \%$

19362

$100 \%$

\section{Season of appointment}

Spring

Summer

Autumn

Winter

3352

4018

7051

4941

19362

${ }^{\mathrm{a}}$ Defined for this data set as: women whose last screen dates were more than 1500 days back (1586 women), incident women with missing date of previous screen (230), or prevalent women who were $\geqslant 53$ years ( 1894 women).

Figure $2 \mathrm{~B}$ shows the formal results for the secondary end point, attendance at initial appointment offered. Attendance was significantly lower for those offered an evening or weekend 
Table 2. Attendance within 120 days (primary end point), attendance at first appointment offered (secondary end point) and number changing between office hours and out of hours appointments

\begin{tabular}{|c|c|c|c|c|}
\hline Trial arm & $\begin{array}{l}\text { Primary end point: } \\
\text { attendance }\end{array}$ & $\begin{array}{l}\text { Secondary end point: attendance at first } \\
\text { appointment offered }\end{array}$ & $\begin{array}{l}\text { Change to } \\
\text { office hours }\end{array}$ & $\begin{array}{c}\text { Change to } \\
\text { OOH }\end{array}$ \\
\hline \multicolumn{5}{|l|}{ Office hours } \\
\hline $\begin{array}{l}\text { Standard }(n=9410) \\
\text { With option to OOH }(n=3510) \\
\text { Sum }(n=12929)\end{array}$ & $\begin{array}{l}6900(73.3 \%) \\
2678(76.1 \%) \\
9578(74.1 \%)\end{array}$ & $\begin{array}{l}4176(44.4 \%) \\
1599(45.4 \%) \\
5775(44.7 \%)\end{array}$ & $\begin{array}{l}- \\
- \\
-\end{array}$ & $\begin{array}{l}157(2.3 \%) \\
194(7.2 \%) \\
351(3.7 \%)\end{array}$ \\
\hline \multicolumn{5}{|l|}{$\mathrm{OOH}$} \\
\hline $\begin{array}{l}\text { Evening }(n=3271) \\
\text { Weekend }(n=3162) \\
\text { Sum }(n=6433)\end{array}$ & $\begin{array}{l}2445(74.8 \%) \\
2295(72.6 \%) \\
4740(73.7 \%)\end{array}$ & $\begin{array}{l}1231(37.6 \%) \\
1375(43.5 \%) \\
2606(40.5 \%)\end{array}$ & $\begin{array}{r}907(37.1 \%) \\
572(24.9 \%) \\
1479(31.2 \%)\end{array}$ & $\begin{array}{l}- \\
- \\
-\end{array}$ \\
\hline \multicolumn{5}{|l|}{ All arms } \\
\hline Total $(n=19362)$ & $14318(73.9 \%)$ & $8381(43.3 \%)$ & & \\
\hline
\end{tabular}

A

Comparisons

Out-of-hours $(\mathrm{OOH})$ vs office hour (major arms) Office hours with or without option Evening / to $\mathrm{OOH}^{*}$ weekend

Trial arms vs standard office hour Standard office Office hours hours (no option with option to $\mathrm{OOH})^{*}$ to $\mathrm{OOH}$

Evening

Weekend

Out-of-hour appointments

Evening*

Weekend

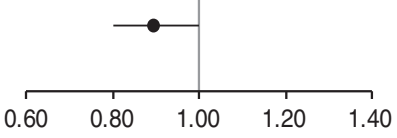

B

Comparisons

Out-of-hours $(\mathrm{OOH})$ vs office hour (major arms)

Office hours with

or without option Evening

$\mathrm{OOH}^{*}$ weekend weekend

Trial arms vs standard office hour Standard office Office hours hours (no option with option to $\mathrm{OOH})^{*}$

to $\mathrm{OOH}$

Evening

Weekend

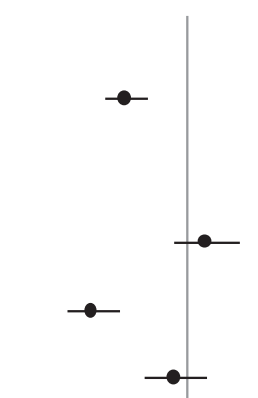

Odds ratio $(95 \% \mathrm{Cl}) \quad P$-value

$0.844(0.794-0.896) \quad<0.001$

$1.044(0.966-1.128) \quad 0.280$

$0.756(0.697-0.821) \quad<0.001$

$0.964(0.889-1.046) \quad 0.382$

Out-of-hour appointments

Evening* Weekend

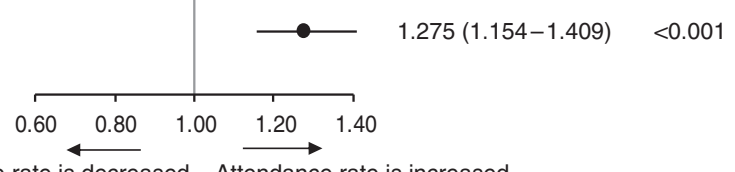

Attendance rate is decreased Attendance rate is increased

*Reference category in logistic regression analysis

Figure 2. Formal trial comparisons. (A) For attendance within 120 days of initial appointment (primary end point). (B) For attendance at initial offered appointment (secondary end point). 
appointment $(40.5 \%$ vs $44.7 \%, \mathrm{OR}=0.844, P<0.001)$. When comparing the three study arms with the standard office hour appointment arm, attendance at initial appointment was significantly lower for evening appointments $(\mathrm{OR}=0.756, P<0.001)$ and slightly lower for Saturday appointments. Within those offered an initial out-of-hours appointment, attendance at weekend appointments was significantly higher $(43.5 \%$ vs $37.6 \%$, OR $=1.275$, $P<0.001$ )

In subgroup analyses, significant heterogeneity of the comparison of the two major arms was observed by prevalent/incident status $(P=0.042)$ and season of appointment $(P<0.001)$ (Supplementary Table 1). Attendance within 120 days (Supplementary Table 2) was particularly low for initial office hour appointments for prevalence episodes (53.6\%) and particularly high for initial office hour appointments for incidence screens $(82.1 \%)$. Attendance was significantly lower for out-of-hours appointments than for office hours appointments in summer (71.3\% vs $76.1 \%, \mathrm{OR}=0.779, P=0.001)$, but significantly higher in spring $(79.9 \%$ vs $76.6 \%, \mathrm{OR}=1.215, P=0.041)$ and autumn $(71.0 \%$ vs $68.7 \%, \mathrm{OR}=1.116, P=0.037)$. Attendance was $77 \%$ for both major arms in winter. There was no significant difference between initial out-of-hours compared with office hour appointments for the four age groups analysed.

For comparisons of minor arms, significant heterogeneity of the difference between office hour appointments and office hour appointments with the option to change was observed by whether the screening unit was static or mobile $(P=0.010)$ and season of appointment $(P<0.001)$. Attendance rates for the subgroups are shown in Table 3 . For static units, higher attendance within 120 days was observed in the option to change group (76.4\% vs $73.2 \%)$, whereas for mobile units, higher attendance was observed in the standard office hour invitation group (77.1\% vs 69.1\%). The option to change group showed higher attendance in summer $(80.5 \%$ vs $75 \%)$ and autumn $(74.1 \%$ vs $66.5 \%)$, but lower attendance in spring (75.2\% vs $77.6 \%)$ and winter $(77.3 \%$ vs $78.0 \%)$. The option to change was associated with a slightly higher attendance in those who had not attended the previous invitation (34.6\% vs 30.6\%), but the interaction did not reach statistical significance $(P=0.246)$. Also, there was no significant heterogeneity by age, but the increased attendance with the option to change was more pronounced in older invitees. The option to change group showed significantly higher attendance in 59 to $<65$ year olds $(79.0 \% v s$ $73.7 \%$ ) and 65 to $<74$ year old women $(76.0 \%$ vs $72.2 \%)$. The heterogeneity of the effect by age, however, was not significant $(P=0.098)$, so this may be a chance finding. No significant heterogeneity was observed for the difference between the initial weekday evening and initial weekend appointment arms.

The majority of reasons for rescheduling of the first-allocated appointment fell into the catch-all category of 'inconvenient' ranging from $81.8 \%$ for the first-allocated evening to $86.9 \%$ for the first-allocated weekend appointments (Supplementary Table 3). The differences in reasons for rescheduling among the arms are significant $(P<0.001)$, mainly due to fewer women allocated to weekend or evening appointments citing 'work' or 'other'.

\section{DISCUSSION}

The results of this randomised trial are very clear. First, the offer of an initial weekend or weekday evening breast screening appointment did not increase attendance rates. However, inclusion of the option to change to such an appointment in the letter inviting women to an office hour appointment was associated with a significant increase in attendance $(76.1 \%$ vs $73.3 \%)$. Around $7 \%$ of those women who were invited took up the option to change, being split approximately equally between changing to evening and

\begin{tabular}{|c|c|c|c|c|}
\hline Category & $\begin{array}{l}\text { Trial arm office } \\
\text { hours }\end{array}$ & Invited & Attended & $\begin{array}{c}\% \\
\text { Attended }\end{array}$ \\
\hline \multicolumn{5}{|c|}{ Screening unit } \\
\hline $\begin{array}{l}\text { Static } \\
\text { Mobile }\end{array}$ & $\begin{array}{l}\text { Standard } \\
\text { With option to } \mathrm{OOH} \\
\text { Standard } \\
\text { With option to } \mathrm{OOH}\end{array}$ & $\begin{array}{c}9004 \\
3383 \\
406 \\
136\end{array}$ & $\begin{array}{c}6587 \\
2584 \\
313 \\
94\end{array}$ & $\begin{array}{l}73.2 \% \\
76.4 \% \\
77.1 \% \\
69.1 \%\end{array}$ \\
\hline \multicolumn{5}{|l|}{ Season } \\
\hline $\begin{array}{l}\text { Spring } \\
\text { Summer } \\
\text { Autumn } \\
\text { Winter }\end{array}$ & $\begin{array}{l}\text { Standard } \\
\text { With option to } \mathrm{OOH} \\
\text { Standard } \\
\text { With option to } \mathrm{OOH} \\
\text { Standard } \\
\text { With option to } \mathrm{OOH} \\
\text { Standard } \\
\text { With option to } \mathrm{OOH}\end{array}$ & $\begin{array}{c}1438 \\
992 \\
2211 \\
570 \\
2930 \\
1235 \\
2831 \\
722 \\
\end{array}$ & $\begin{array}{c}1116 \\
746 \\
1658 \\
459 \\
1947 \\
915 \\
2179 \\
558\end{array}$ & $\begin{array}{l}77.6 \% \\
75.2 \% \\
75.0 \% \\
80.5 \% \\
66.5 \% \\
74.1 \% \\
77.0 \% \\
77.3 \%\end{array}$ \\
\hline \multicolumn{5}{|c|}{ Attendance at previous screen } \\
\hline $\begin{array}{l}\text { Attended } \\
\text { Not-attended }\end{array}$ & $\begin{array}{l}\text { Standard } \\
\text { With option to } \mathrm{OOH} \\
\text { Standard } \\
\text { With option to } \mathrm{OOH}\end{array}$ & $\begin{array}{c}5700 \\
2394 \\
1933 \\
650\end{array}$ & $\begin{array}{c}5003 \\
2112 \\
591 \\
225\end{array}$ & $\begin{array}{l}87.8 \% \\
88.2 \% \\
30.6 \% \\
34.6 \%\end{array}$ \\
\hline \multicolumn{5}{|l|}{ Age group } \\
\hline 47 to $<54$ & $\begin{array}{l}\text { Standard } \\
\text { With option to } \mathrm{OOH}\end{array}$ & $\begin{array}{c}2332 \\
732\end{array}$ & $\begin{array}{c}1713 \\
541\end{array}$ & $\begin{array}{l}73.5 \% \\
73.9 \%\end{array}$ \\
\hline 54 to $<59$ & $\begin{array}{l}\text { Standard } \\
\text { With option to } \mathrm{OOH}\end{array}$ & $\begin{array}{c}2191 \\
676\end{array}$ & $\begin{array}{c}1621 \\
503\end{array}$ & $\begin{array}{l}74.0 \% \\
74.4 \%\end{array}$ \\
\hline 59 to $<65$ & $\begin{array}{l}\text { Standard } \\
\text { With option to } \mathrm{OOH}\end{array}$ & $\begin{array}{c}2563 \\
972\end{array}$ & $\begin{array}{c}1889 \\
768\end{array}$ & $\begin{array}{l}73.7 \% \\
79.0 \%\end{array}$ \\
\hline 65 to $<74$ & $\begin{array}{l}\text { Standard } \\
\text { With option to } \mathrm{OOH}\end{array}$ & $\begin{array}{l}2324 \\
1139\end{array}$ & $\begin{array}{c}1677 \\
866\end{array}$ & $\begin{array}{l}72.2 \% \\
76.0 \%\end{array}$ \\
\hline
\end{tabular}

weekend appointments. Although the comparison of the major trial arms suggests that initial office hour appointments are preferred, the minority for whom the opportunity to change to out-of-hours appointments is attractive is of sufficient size to confer a significant improvement.

Subgroup analyses suggest that the initial office hour appointment results in particularly high attendance at incident screen episodes, that is, in women who have attended for screening in the past. However, the increased attendance with an office hour appointment with the option to change was slightly stronger in previous non-attenders. A number of barriers to breast cancer screening, including time and employer constraints, have been indentified (Schueler et al, 2008). A recent population-based survey in United Kingdom found that $12.5 \%$ of women, who had never attended breast screening, cited '...I haven't got round to it but I do intend to take part' (Lo et al, 2013). Intention to attend screening has been considered to be a potentially important variable, and women who had a positive attitude towards screening were more likely to attend in a number of studies (Jepson et al, 2000). The option to change to more convenient appointment times, which fit around work commitments or other time constraints, may reduce these barriers to screening attendance for women with the intention to attend but who have in the past failed to translate this intention into behaviour. 
The finding of a preference for office hour initial appointments but an increased uptake with the opportunity to change was stronger in the summer months. Screening appointments in the evenings might be more attractive in summer months because of the longer daylight hours and appointments on Saturdays as women might be more inclined to combine their screening appointment with a social occasion or shopping in town when the weather is more pleasant. Women who attended Saturday appointments at the centrally located Avon Breast Screening Programme in Bristol commented that they like to combine their Saturday appointment with shopping in the close-by shopping area.

Female employment rates are now higher than in the past (66\% in 2012 compared with 62\% in 1992 for the age group 16-64) (Office for National Statistics, 2013). With the biggest increase in employment occurring in the age group 50-64 (47\% in 1992 to $61 \%$ in 2012) and the age group 35-49 having the highest employment rates ( $73 \%$ in 1992 to $76 \%$ in 2012), one might assume that younger women (age groups 47 to $<54$ and 54 to $<59$ in this study) would be more likely to be in full time work. We would have therefore expected that out-of-hours appointments would be more attractive to younger women. However, we observed that increased attendance with the option to change was more pronounced in older invitees (aged $\geqslant 59$ years). The heterogeneity of the effect by age was not significant, however, so this may be a chance finding.

A study in the 1990s in one of the centres used here found that the offer of a Saturday appointment did not improve attendance in women who had not attended their initial appointment (Readman and Asbury, 1999). The present trial differs in the interventions assessed and was aimed at the entire invited population. Also, it might be that changes in female employment patterns have rendered the option to change more attractive.

In conclusion, the findings of this trial are very clear: the optimum strategy is to offer an initial office hour breast screening appointment, with the offer of the opportunity to change to a weekend appointment. Service managers should plan for around $7 \%$ of the invited population changing to an evening or weekend appointment, in roughly equal proportions.

\section{ACKNOWLEDGEMENTS}

This trial was supported by the NHS Cancer Screening Programmes. SWD and DP contributed to this work as part of the programme of the Policy Research Unit in Cancer Awareness, Screening and Early Diagnosis. The Policy Research Unit in Cancer Awareness, Screening, and Early Diagnosis receives funding for a research programme from the Department of Health Policy Research Programme. It is a collaboration between researchers from seven institutions (Queen Mary University of London, UCL, King's College London, London School of Hygiene and Tropical Medicine, Hull York Medical School, Durham University, and Peninsula Medical School). We would like to thank the staff at the
Nightingale Centre and Genesis Prevention Centre and the Avon Breast Screening Unit for their contribution to this study.

CONFLICT OF INTEREST

The authors declare no conflict of interest.

\section{REFERENCES}

Alcaraz M, Lluch A, Miranda J, Pereiro I, Salas MD (2002) [Study of non-participation in the breast cancer screening program in the city of Valencia (Spain)]. Gac Sanit 16: 230-235.

Breslow NE, Day NE (1980) Statistical methods in cancer research. Volume I-The analysis of case-control studies. IARC 1: 5-338.

Engelman KK, Cizik AM, Ellerbeck EF (2005) Women's satisfaction with their mammography experience: results of a qualitative study. Women Health 42: 17-35.

Gail MH, Tan WY, Piantadosi S (1988) Tests for no treatment effect in randomized clinical trials. Biometrika 75: 57-64.

Independent UK Panel on Breast Cancer Screening (2012) The benefits and harms of breast cancer screening: an independent review. Lancet 380: 1778-1786.

Jepson R, Clegg A, Forbes C, Lewis R, Sowden A, Kleijnen J (2000) The determinants of screening uptake and interventions for increasing uptake: a systematic review. Health Technol Assess 4: i-vii 1-133.

Lo SH, Waller J, Wardle J, von Wagner C (2013) Comparing barriers to colorectal cancer screening with barriers to breast and cervical screening: a population-based survey of screening-age women in Great Britain. J Med Screen; doi:10.1177/0969141313492508.

Maheswaran R, Pearson T, Jordan H, Black D (2006) Socioeconomic deprivation, travel distance, location of service, and uptake of breast cancer screening in North Derbyshire, UK. J Epidemiol Commun H 60 : 208-212.

NHS Cancer Screening Programmes (2011) NHS Breast Screening Programme: Annual Review 2011, Sheffield.

Office for National Statistics (2013) Labor Force Survey.

Paskett E, Tatum C, Rushing J, Michielutte R, Bell R, Long Foley K, Bittoni M, Dickinson SL, McAlearney AS, Reeves K (2006) Randomized trial of an intervention to improve mammography utilization among a triracial rural population of women. J Natl Cancer I 98: 1226-1237.

Readman LP, Asbury DL (1999) Breast screening uptake rates: does access to Saturday sessions lead to improvements? Breast 8: 343-344.

Richardson A (1990) Factors likely to affect participation in mammographic screening. N Z Med J 103: 155-156.

Rutter DR, Steadman L, Quine L (2006) An implementation intentions intervention to increase uptake of mammography. Ann Behav Med 32: 127-134.

Schueler KM, Chu PW, Smith-Bindman R (2008) Factors associated with mammography utilization: a systematic quantitative review of the literature. J Womens Health (Larchmt) 17: 1477-1498.

This work is published under the standard license to publish agreement. After 12 months the work will become freely available and the license terms will switch to a Creative Commons AttributionNonCommercial-Share Alike 3.0 Unported License.

Supplementary Information accompanies this paper on British Journal of Cancer website (http://www.nature.com/bjc) 